\title{
White Matter Microstructural Abnormality in Children with Hydrocephalus Detected by Probabilistic Diffusion Tractography
}

A. Rajagopal, J.S. Shimony, R.C. McKinstry, M. Altaye, T. Maloney, F.T. Mangano, D.D. Limbrick, S.K. Holland, B.V. Jones, S. Simpson,

D. Mercer, and W. Yuan

\begin{abstract}
BACKGROUND AND PURPOSE: Hydrocephalus is a severe pathologic condition in which WM damage is a major factor associated with poor outcomes. The goal of the study was to investigate tract-based WM connectivity and DTI measurements in children with hydrocephalus by using the probabilistic diffusion tractography method.

MATERIALS AND METHODS: Twelve children with hydrocephalus and 16 age-matched controls were included in the study. Probabilistic diffusion tractography was conducted to generate tract-based connectivity distribution and DTI measures for the genu of the corpus callosum and the connectivity index. Tract-based summary measurements, including the connectivity index and DTI measures (fractional anisotropy, mean diffusivity, axial diffusivity, and radial diffusivity), were calculated and compared between the 2 study groups.
\end{abstract}

RESULTS: Tract-based summary measurement showed a higher percentage of voxels with lower normalized connectivity index values in the WM tracts in children with hydrocephalus. In the genu of the corpus callosum, the left midsegment of the corticospinal tract, and the right midsegment of the corticospinal tract, the normalized connectivity index value in children with hydrocephalus was found to be significantly lower $(P<.05$, corrected). The tract-based DTI measures showed that the children with hydrocephalus had significantly higher mean diffusivity, axial diffusivity, and radial diffusivity in the genu of the corpus callosum, left midsegment of the corticospinal tract, and right midsegment of corticospinal tract and lower fractional anisotropy in the genu of the corpus callosum $(P<.05$, corrected).

CONCLUSIONS: The analysis of WM connectivity showed that the probabilistic diffusion tractography method is a sensitive tool to detect the decreased continuity in WM tracts that are under the direct influence of mechanical distortion and increased intracranial pressure in hydrocephalus. This voxel-based connectivity method can provide quantitative information complementary to the standard DTI summary measures.

ABBREVIATIONS: $\mathrm{AD}=$ axial diffusivity $; \mathrm{Cl}=$ connectivity index; $\mathrm{FA}=$ fractional anisotropy $\mathrm{CC}=$ corpus callosum; $\mathrm{mCST}=$ midsegment of corticospinal tract; $M D=$ mean diffusivity; $P D T=$ probabilistic diffusion tractography; $R D=$ radial diffusivity

$\mathbf{P}$ ediatric hydrocephalus is a common medical condition characterized by an imbalance in CSF production and absorption, leading to elevated intracranial pressure and ventriculomegaly. Previous studies of hydrocephalus have consistently found functional deficits in a series of neuropsychological domains, including visuospatial, motor, ${ }^{1}$ and nonverbal learning, ${ }^{2}$ presumably resulting from white matter damage or degeneration. ${ }^{3}$

DTI is a noninvasive MR imaging technique that quantifies diffusion properties of in vivo brain structures, especially in the

Received December 13, 2012; accepted after revision March 11, 2013.

From the Pediatric Neuroimaging Research Consortium (A.R., T.M., S.K.H., B.V.J., S.S., W.Y.), Department of Radiology, Division of Biostatistics and Epidemiology (M.A.), and Division of Pediatric Neurosurgery (F.T.M.), Cincinnati Children's Hospital Medical Center, Cincinnati, Ohio; University of Cincinnati College of Medicine (M.A. F.T.M., S.K.H., B.V.J., W.Y.), Cincinnati, Ohio; Mallinckrodt Institute of Radiology (J.S.S., R.C.M.) and Department of Neurological Surgery (D.D.L., D.M.), Washington University School of Medicine, St. Louis, Missouri.

This work was supported by a grant from NIH/NINDS (1R01 NS066932).

The data reported in this manuscript have not been published elsewhere. white matter. Fractional anisotropy (FA) values allow us to infer WM integrity, or the lack thereof, for underlying pathologic changes in myelin sheaths, axonal membranes, and intercellular/ extracellular spaces. Past DTI studies in hydrocephalus have identified structural damage in the corpus callosum and internal capsule on the basis of abnormal diffusion properties in these important WM structures. ${ }^{4,5}$

The severe deformation in the hydrocephalic brain presents a unique challenge for imaging data processing and analysis. Past DTI studies of pediatric hydrocephalus have been based on region-of-interest analysis. ${ }^{1,6}$ Although region of interest-based analysis has helped demonstrate evidence of structural abnormalities in key WM regions, there are limitations inherent in this type

\footnotetext{
Please address correspondence to Weihong Yuan, PhD, Pediatric Neuroimaging Research Consortium, Department of Radiology, Cincinnati Children's Hospital, 3333 Burnet Ave, Cincinnati, OH 45229; e-mail: Weihong.Yuan@cchmc.org

- Indicates open access to non-subscribers at www.ajnr.org

http://dx.doi.org/10.3174/ajnr.A3737
}

AJNR Am J Neuroradiol 34:2379-85 Dec 2013 www.ajnr.org 
of analysis: The delineation of the region of interest is limited to 1 or a small number of sections, usually subjectively selected, that may or may not lead to a full representation of the WM structure of interest. There have been new developments in image registration methods that have shown initial success in their applications in brains with large ventricles and/or distortion ${ }^{7-9}$; however, they are not tailored for application in the pediatric hydrocephalic brains with severe distortion, especially in children at a very young age.

The aim of the present study was to explore the feasibility of applying probabilistic diffusion tractography (PDT) in children with hydrocephalus to quantify structural integrity in a series of WM fiber tracts, including the genu of the corpus callosum (CC), splenium of the CC, and midsegment of corticospinal tract $(\mathrm{mCST})$. The connectivity index (CI) (a measure of WM connectivity between 2 brain regions) was calculated for each voxel as the sum of the number of streamlines that started from the seed region, passed through the voxel, and reached the target region. The deviation of the CI from the normal range may indicate compromised integrity in the myelin sheath and axons under pathologic conditions. We examined the tract-based CI values and DTI values and explored the complementary aspect of the CI and FA in differentiating the hydrocephalus group from controls.

\section{MATERIALS AND METHODS}

\section{Subjects}

Twelve children (age range, 0 -38.5 months) who were diagnosed with hydrocephalus and referred for surgical treatment were included in the present study. An age-matched control group $(n=$ 16 ; age range, $0.6-37.5$ months) was also included for comparison. All participants were recruited for a multicenter study from either the Cincinnati Children's Hospital Medical Center (7 patients with hydrocephalus, 15 controls) or St. Louis Children's Hospital (5 patients with hydrocephalus, 1 control). The inclusion/exclusion criteria have been described elsewhere. ${ }^{1}$ Both local institutional review board committees approved the study protocol, and written informed consent was obtained from parents of the participants.

\section{DTI Data Acquisition}

DTI data were acquired at both sites during the clinical scans by using a 1.5T MR imaging system (either Signa; GE Healthcare, Milwaukee, Wisconsin or Avanto; Siemens, Erlangen, Germany). The imaging protocol included a single-shot EPI diffusionweighted spin-echo sequence $(\mathrm{FOV}=240 \times 240 \mathrm{~mm}$; matrix $=$ $96 \times 96$; resolution $=2.5 \mathrm{~mm}$ isotropic; $\mathrm{TR} / \mathrm{TE}=9400 / 93.2 \mathrm{~ms}$; $\mathrm{NEX}=2)$ and $\mathrm{a} 3 \mathrm{D}$ anatomic $\mathrm{T} 1$ sequence $(\mathrm{FOV}=256 \times 256$ $\mathrm{mm}$; matrix $=256 \times 256$; resolution $=1 \mathrm{~mm}$ isotropic). Diffusion-weighting was applied along 15 directions with a b-value of $1000 \mathrm{~s} / \mathrm{mm}^{2}$, and 1 additional image was acquired with no diffusion-weighting ( $\mathrm{b}$-value $=0 \mathrm{~s} / \mathrm{mm}^{2}$ ). Siemens gradient weightings were programmed to match the implementation on the GE Healthcare scanner. The compatibility of MR imaging data acquired from different scanners at 2 different research sites was established initially by using an MR phantom (BIRN: Biomedical Informatics Research Network; http://www.nbirn.net) and a human phantom and then maintained annually. The interscanner difference was minimal $(<1.1 \%)$ on the basis of mean diffusivity (MD) values measured from the BIRN phantom. The relative interscanner difference based on the human phantom was $<3.3 \%$ and 3.5\% for FA and MD, respectively. The details of the quality assurance procedure have been reported in previous work. ${ }^{1,10}$

\section{DTI Data Processing and Probabilistic Diffusion Tractography}

Image processing and analysis were performed by using the fMRI of the Brain Software Library (FSL; http://www.fmrib.ox.ac.uk/ fsl). DTI data were preprocessed with eddy current correction and minor head motion by using affine registration to a reference volume. The preprocessed images were then fit to a diffusion tensor model at each voxel. Summary measures, including FA, MD, axial diffusivity $(\mathrm{AD})$, and radial diffusivity $(\mathrm{RD})$, were calculated on the basis of standard methods.

At each voxel, the Bayesian Estimation of Diffusion Parameters Obtained by using Sampling Techniques (BEDPOSTX) function in FSL was used to build up the distributions on diffusion parameters on the basis of the Markov Chain Monte Carlo sampling. ${ }^{11}$ The outputs of the BEDPOSTX were used to compute the PDT by using Probtrackx ${ }^{12}$ in FSL. The seed and target masks used in the PDT for genu of the CC were created on sagittal sections as illustrated in Fig 1. The 2 masks for the left or right mCST were created on axial sections placed between 1 section below the body of the corpus callosum and the entry of the posterior limb of the internal capsule to the cerebral peduncle (Fig 2). All masks were delineated by 1 operator (W.Y.) and confirmed by 3 pediatric radiologists (R.C.M., J.S.S., and B.V.J.) and 2 pediatric neurosurgeons (F.T.M., D.D.L.).

CI was extracted for all the voxels from the PDT-derived WM tracts. The fiber tracking drew 5000 samples from each voxel in the seed mask. The CI value for any voxel in the brain is the sum of all the tracking samples that originated from the seed mask, passed through the voxel, and reached the target region. Only the voxels between the 2 masks were retained for analysis. A threshold of 100 was used for the connectivity index to avoid spurious results as the consequence of partial volume effect and image noise. ${ }^{13,14}$ We also tested more stringent threshold levels at 150 and 200, and the findings remained the same. Therefore, only the data based on a threshold of 100 are reported in the present study.

\section{Data Analysis}

The comparison between the children with hydrocephalus and the control group was made in 4 PDT-derived WM tracts (ie, the genu of the CC, splenium of the CC, left mCST, and right mCST). The fiber tracking was successful in all participants in the control group for all the WM tracts. It was also successful in the hydrocephalus group for the genu of the CC, left mCST, and right mCST (successful rates were 11/12, 12/12, 12/12 for the 3 tracts, respectively). However, fiber tracking was not successful in the splenium of the CC in 9/12 children with hydrocephalus. Therefore, the data for the splenium of the CC were not included in the eventual analysis. This outcome may be due to the expansion of ventricle volume that caused the stretching of the occipital horn of the ventricles, leading to a sharp bend in the splenium of the CC.

Histograms of the distribution of CI values within a tract showed that they did not follow normal distribution. To quantify the degree of skewness for the within-tract CI and DTI measures, we used the following index: Skewness $=\mu_{3} / \delta^{3}$, where $\mu$ is the mean, $\mu_{3}$ represents the third moment about the mean $\mu$, and $\delta$ is 

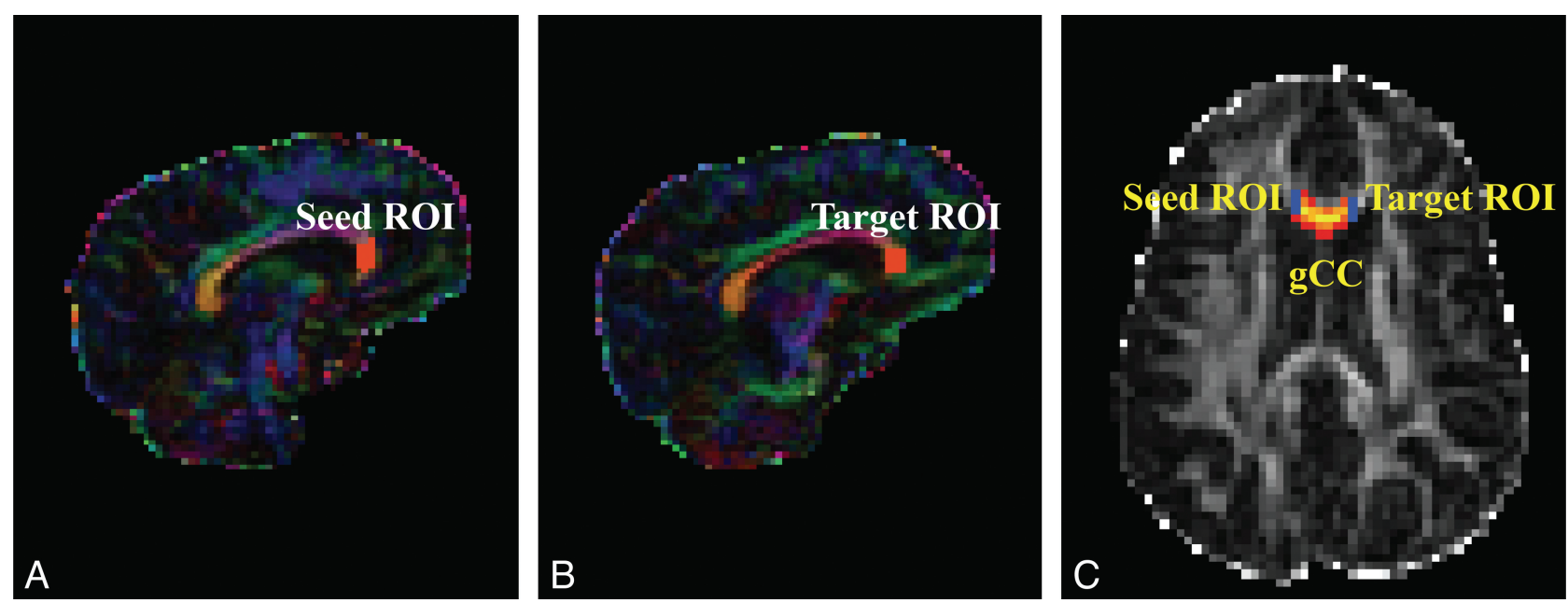

FIG 1. DTI maps demonstrating seed and target masks and resultant probabilistic fiber tracking of the genu of the CC in representative participants. $A-C$, hydrocephalus. $A$ and $B$, Color-coded FA maps. C, FA map. The masks shown in orange in the sagittal maps can be seen as short blue lines on axial map.
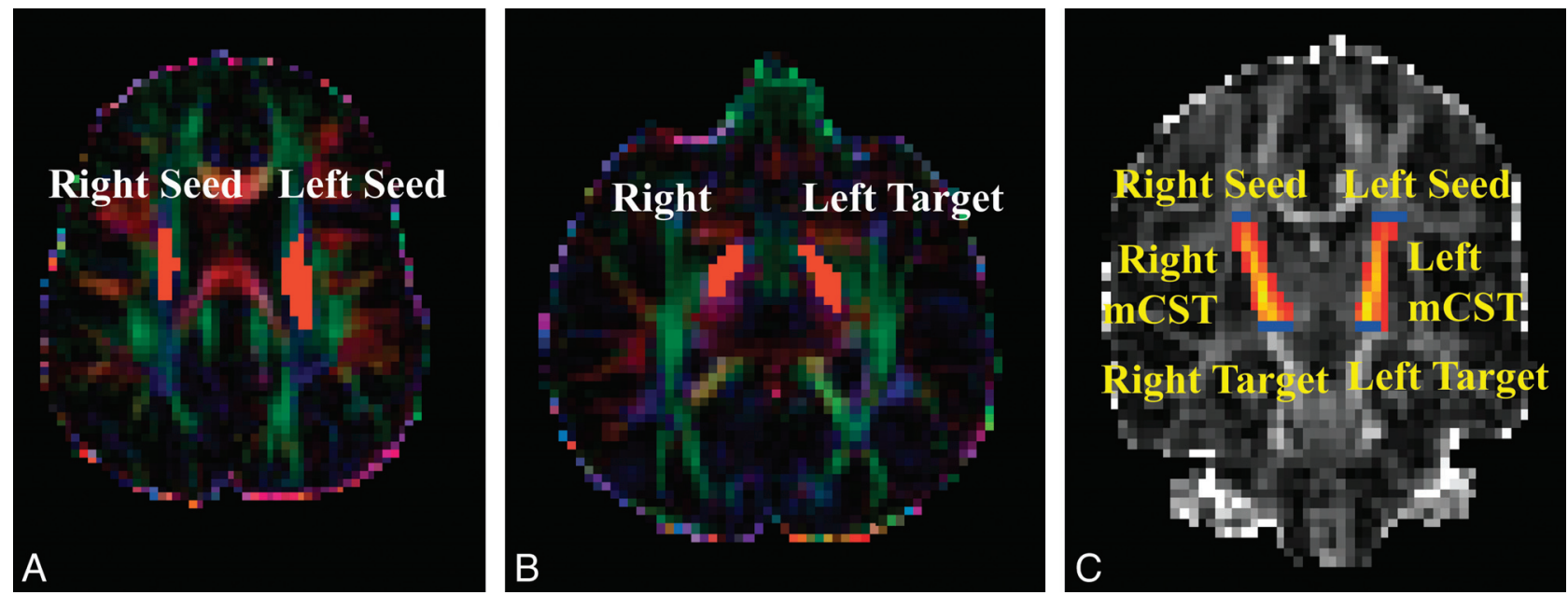

FIG 2. DTI maps demonstrating seed and target masks and resultant probabilistic fiber tracking of the left and right midsegments of the cortical spinal tracts in representative participants. $A-C$, Hydrocephalus. $A$ and $B$, Color-coded FA maps. $C$, FA map. The masks shown in orange on the axial DTI maps can be seen as short blue lines on coronal FA map.

the SD. A skew to the left or right direction of a distribution was called a positive or negative skew, respectively. The skewness index was calculated for each tract in individual subjects first and then for group comparison.

Due to the age range of the study population and the expected developmental change in brain structures, it was not feasible to maintain the size and shape of the seed or target masks as constant across all subjects in the fiber tracking. Initial correlation analysis showed that in some cases the seed and target mask, as well as the tract volume, were significantly correlated with the median CI value from all the voxels within the tract. Thus, a new index, normalized CI (CI/tract volume, units of samples/voxel), was used in the study for group comparison. The tract volume (the number of voxels in the tract) is, in theory, affected by both seed and target mask size (as confirmed by our initial data). The drawing of these masks can also be affected by the brain tissue area, which is age-related, and are operator-dependent. Using the tract volume as the weighting factor in the index of normalized CI minimizes the above-mentioned factors.

\section{Statistical Analysis}

The group difference of the skewness index was tested by using the Mann-Whitney $U$ test. Multiple regression analysis was performed by using either the DTI measurement (FA, MD, AD, or $\mathrm{RD}$ ) or the normalized CI as a dependent variable and group and age as independent variables. Multiple comparisons were corrected by using the false discovery rate method. ${ }^{15}$

\section{RESULTS}

\section{Comparison of Within-Tract Skewness in Connectivity} Index and FA Values

Skewness in CI for each tract is plotted in Fig 3. In all 3 tracts analyzed, CI values within a tract were found to skew to the left of the distribution with a long tail extended in the high CI direction (Fig 3 and Table 1). The CI within-tract skewness in any group/ tract centered between 0.94 and 1.33 , leading to a significant deviation from symmetric distribution in this index $(P<.05$ for all groups). Therefore, the mean of the CI value is not considered a reflection of the CI values across all the voxels within a certain 
tract. For subsequent analysis of CI measures, the 25th, 50th and 75 th percentile values were selected. On the other hand, the skewness for within-tract FA was found to center close to zero; therefore, the mean value was an appropriate summary measure for further analysis and comparison.

No statistically significant group difference was found between the children with hydrocephalus and those in the control group in either CI skewness or FA skewness in any of the 3 tracts analyzed.

\section{Aggregated Normalized Connectivity Index Values in the Genu of the CC, Left mCST, and Right mCST}

Figure 4 shows the percentile histograms of the aggregated normalized CI. In all 3 tracts, children with hydrocephalus were found to have a lower percentage of voxels with high normalized CI values. The percentage of the voxels with normalized CI values higher than 20 (an arbitrary value selected at approximately where the 2 distribution curves cross) in the genu of the CC (Fig 4A) was 30.5\% for children with hydrocephalus and $56.3 \%$ for controls. Similar differences were also found in the left mCST (Fig 4B, 13.1\% versus 27.0\%) and right mCST (Fig $4 C, 8 \%$ versus $27.1 \%$ ).

In the mCST, the distribution curve for the hydrocephalus group skewed more toward the low end, with the tail ending at a value of 40 , while the control still had $6.4 \%$ and $8.4 \%$ of the voxels in the left $\mathrm{mCST}$ and right $\mathrm{mCST}$, respectively, which are higher than 40 in normalized CI values (Fig $4 E,-F$ ).

\section{Tract-Based Summary Connectivity Measures (Normalized Connectivity Index Values)}

As shown in Fig 5 and Table 2, the median within-tract normalized CI values in the patients with hydrocephalus were significantly lower than those in the control group $(P<.05$, corrected) in all $3 \mathrm{WM}$ tracts examined. The difference was also significant $(P<$ .05 , corrected) or at trend level (right mCST in the first quartile of normalized CI) at both the first and the third quartiles of the withintract normalized CI levels. Age was not found to be a significant predictor of normalized CI in any of the analyses.

\section{Tract-Based Summary DTI Measures (Mean FA, MD, AD, and RD Values)}

As shown in Fig 6 and Table 3, on the basis of the multiple regression analysis, age was found to be a factor that affected all the DTI parameters examined in all 3 tracts at a statistically significant level $(P<.05$, corrected) or at least at trend level ( $\mathrm{AD}$ in genu of the $\mathrm{CC}$ ). After age was accounted for, significant group differences of tract-based DTI measurements were found in $\mathrm{MD}, \mathrm{AD}$, and $\mathrm{RD}$ in all $3 \mathrm{WM}$ tracts and the group difference was significant in FA in the genu of the CC (all $P<.05$, corrected).

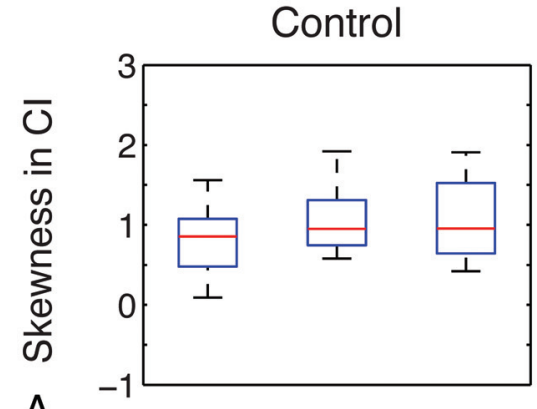

A

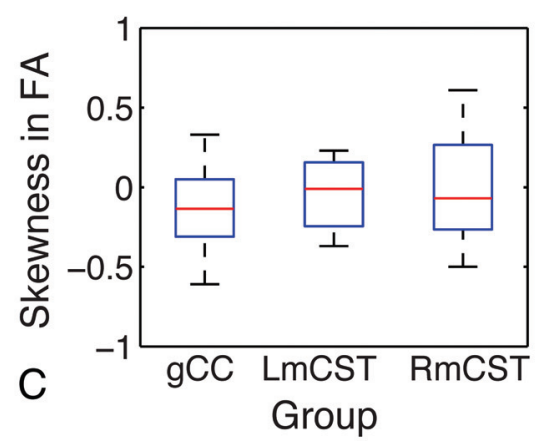

Hydrocephalus
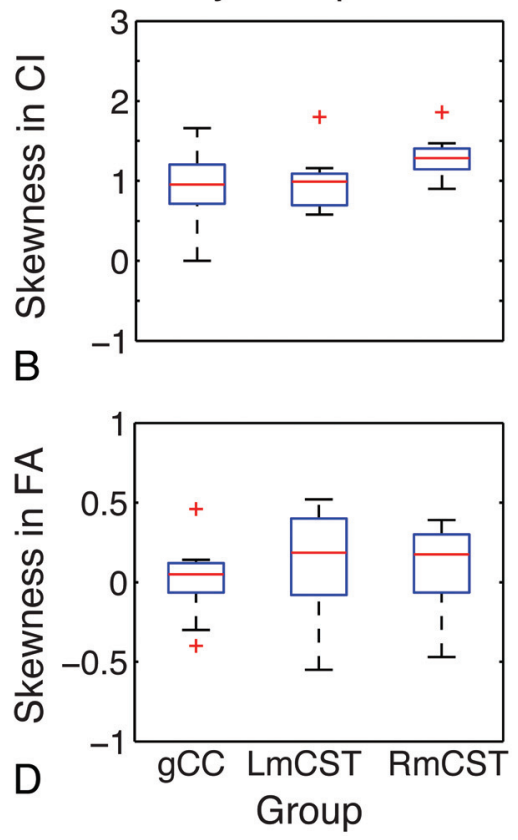

FIG 3. Boxplot showing the skewness index for connectivity index ( $A$ and $B$ ) and FA ( $C$ and $D)$.

\section{DISCUSSION}

Probabilistic tractography is a powerful fiber tracking method that calculates multiple pathways emanating from a seed region of interest and from each point along the reconstructed trajectories. It avoids the use of an FA threshold, thus allowing tracking to continue through brain regions where the level of uncertainty would cause a deterministic algorithm to fail. ${ }^{11,16-18}$ In this study, we investigated tract-based WM connectivity and DTI measurements in children with hydrocephalus by using PDT. We tested and proved the working hypotheses: 1) The connectivity index derived from PDT is decreased in children with hydrocephalus; and 2) DTI measurements derived from PDT are abnormal in children with hydrocephalus, reflecting disruption in fiber continuity.

The most prominent functional deficits that are consistently reported in pediatric

Table 1: Descriptive statistics of the within-tract connectivity index and FA skewness

\begin{tabular}{|c|c|c|c|c|c|c|c|}
\hline \multirow[b]{2}{*}{ Tracts } & \multirow[b]{2}{*}{ Group } & \multicolumn{3}{|c|}{$\mathrm{Cl}$} & \multicolumn{3}{|c|}{ FA } \\
\hline & & Min & Median & Max & Min & Median & Max \\
\hline \multirow[t]{2}{*}{ gCC } & Control $(n=16)$ & 0.2 & 0.94 & 1.56 & -0.61 & -0.13 & 0.33 \\
\hline & Hydrocephalus ( $n=11)$ & 0.44 & 1.04 & 1.66 & -0.4 & 0.1 & 0.46 \\
\hline \multirow[t]{2}{*}{ Left mCST } & Control $(n=16)$ & 0.58 & 0.97 & 1.92 & -0.37 & 0.00 & 0.23 \\
\hline & Hydrocephalus ( $n=12)$ & 0.58 & 1.05 & 1.80 & -0.55 & 0.19 & 0.52 \\
\hline \multirow[t]{2}{*}{ Right mCST } & Control $(n=16)$ & 0.42 & 1.10 & 1.91 & -0.5 & -0.01 & 0.61 \\
\hline & Hydrocephalus ( $n=12)$ & 0.9 & 1.33 & 1.86 & -0.47 & 0.18 & 0.39 \\
\hline
\end{tabular}

Note:-Min indicates minimum; Max, maximum; gCC, genu of the CC. 
patients with hydrocephalus are in such domains as motor, visuospatial, and other nonverbal intelligences. ${ }^{1-3}$ These deficits have been associated with myelin sheath breakdown, axonal degeneration, or even loss of axons in the corpus callosum and periventricular white matter based on both human and animal neuropathologic studies. ${ }^{19}$ In the present study, statistically significant differences in both the summary DTI measures and the connectivity measure were found in the comparison between children with hydrocephalus and those in the control group. We interpret this as evidence of WM changes as the result of neuropathology seen in hydrocephalus.

The connectivity index maps (Figs 1 and 2) are the connectivity distribution maps with the value at each voxel being the sum of the
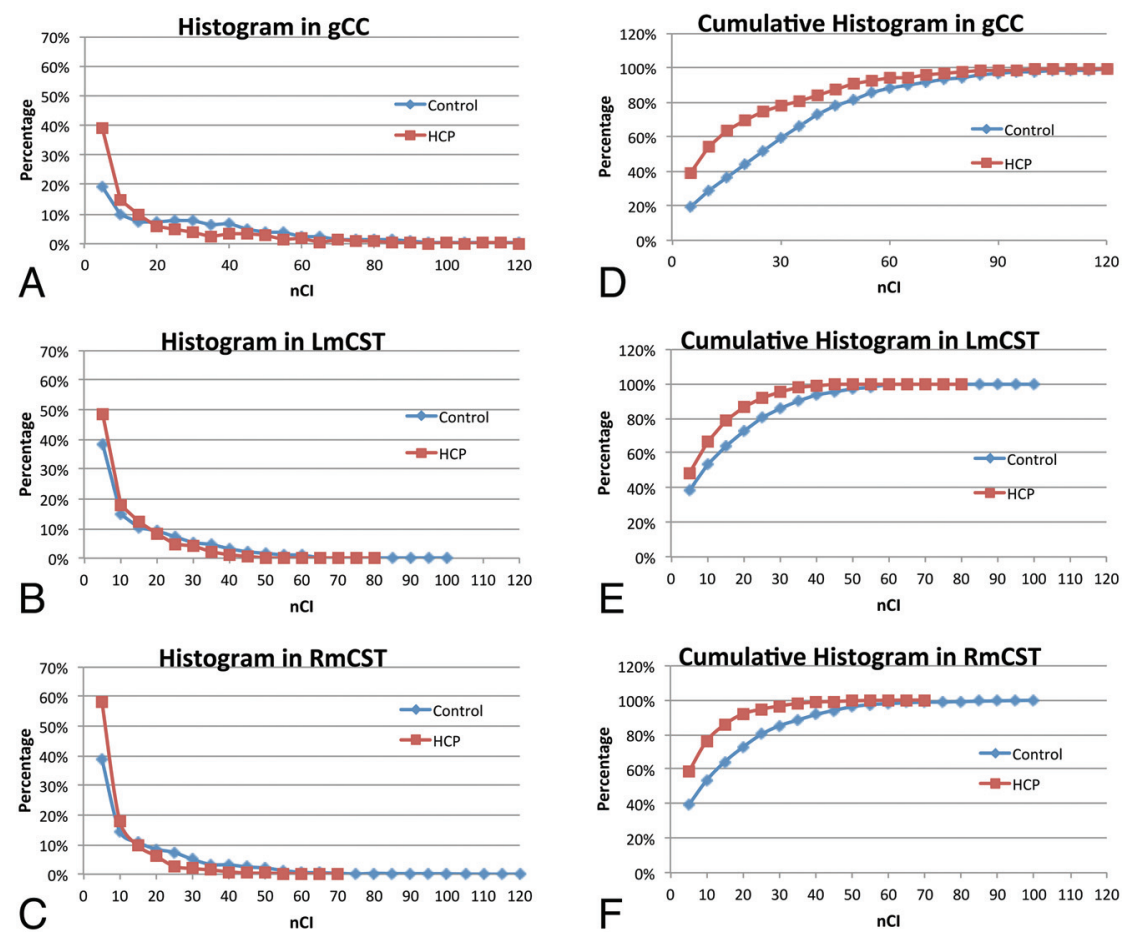

FIG 4. Percentile histograms of the normalized connectivity index $(\mathrm{nCl})$ values for children with hydrocephalus and controls, respectively, in the genu of the CC $(A)(30.5 \%$ and $56.3 \%)$, left mCST (B) (13.1\% and $27.0 \%)$, and right $\mathrm{mCST}$ (C) (8\% and $27.1 \%)$. The corresponding cumulative percentile histograms for the genu of the CC, left $\mathrm{mCST}$, and right $\mathrm{mCST}$ are shown in $D-F$, respectively. sample streamlines passing through the voxel. In the present study, we defined a seed mask and a target mask for each subject on the basis of anatomic landmarks, to restrict the tractography in all the tracts examined to yield the specific tracts of interest. The connectivity measure is a quantification of the strength, or probability, of the connectivity for all those connections initiated from the seed voxels and reaching the target masks. Similar approaches based on PDT have been adopted by other studies to investigate the pathologic progression in amyotrophic lateral sclerosis, ${ }^{14}$ to contrast the organization of prefrontal projection pathways in humans and macaque monkeys. ${ }^{20}$

In the present study, the histogram based on the aggregated normalized CI measures showed that children with hydrocephalus had a lower percentage of voxels with higher connectivity and a higher percentage of voxels with low connectivity in all 3 tracts examined. The comparison of the within-tract summary connectivity measure between the 2 groups showed a consistent significant decrease in normalized CI values in children with hydrocephalus. As expected, examination of the connectivity index maps (Figs 1 and 2 as examples) showed that the voxels with high connectivity were located in the central portion of the tracts, while the voxels at the peripheral areas of a tract tended to have lower connectivity. We examined the summary connectivity measure (normalized CI) not only at the median but also at the lower and upper quartile values. The fact that the group contrast was significant at all 3 quartile levels indicates that this summary connectivity index, normalized CI, is sensitive to the pathology resulting from hydrocephalus throughout different portions of WM tracts in children with hydrocephalus.

Tract-based summary DTI measures showed a significant increase of $\mathrm{MD}, \mathrm{AD}$, and RD values, which was consistent in all
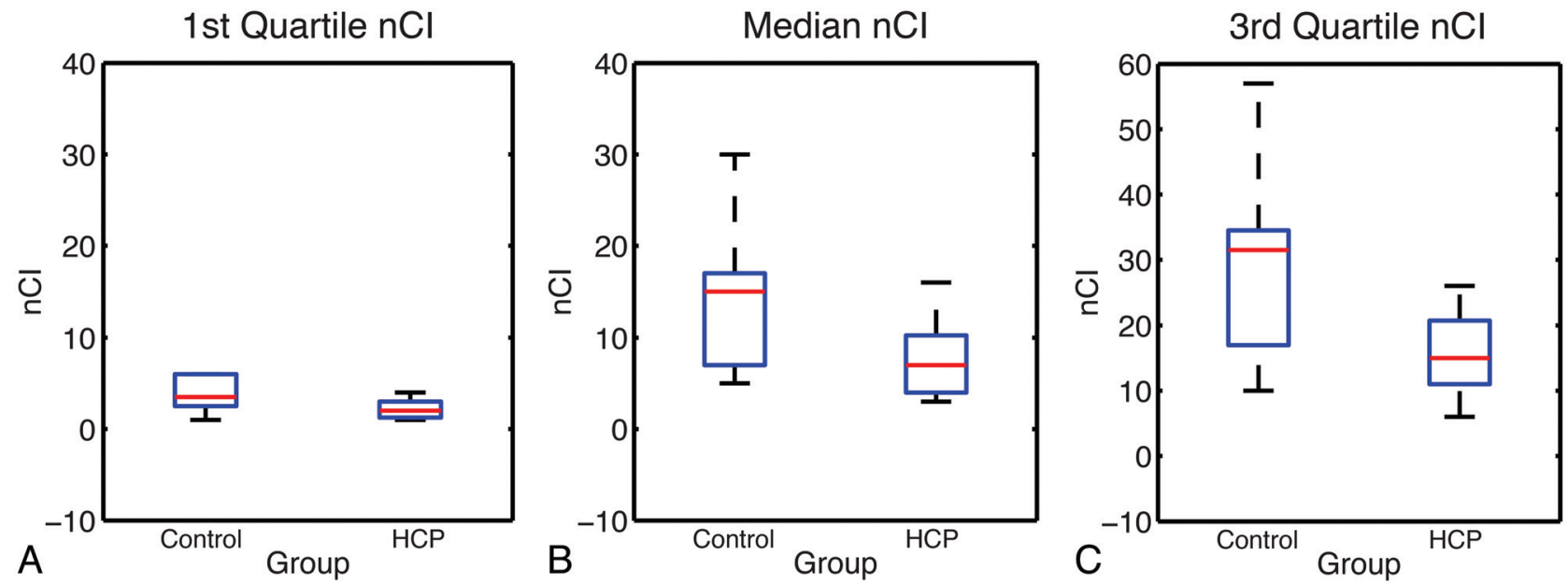

FIG 5. Boxplot showing the normalized $\mathrm{Cl}$ for left mCST by using 3 different quartile levels of the normalized $\mathrm{Cl}$ as a summary measurement. $A$, First quartile. $B$, Median. $C$, Third quartile. 
Table 2: Multiple regression analysis for each tract at 3 quartiles of normalized $\mathrm{Cl}^{\mathrm{a}}$

\begin{tabular}{|c|c|c|c|c|c|c|c|c|c|c|c|}
\hline & \multirow[b]{2}{*}{$d f$} & \multirow{2}{*}{$\begin{array}{c}\text { Dependent } \\
\text { Variables }\end{array}$} & \multicolumn{3}{|c|}{ First Quartile } & \multicolumn{3}{|c|}{ Median } & \multicolumn{3}{|c|}{ Third Quartile } \\
\hline & & & SE & $T$ Value & $P$ & SE & $T$ Value & $P$ & SE & $T$ Value & $P$ \\
\hline \multirow[t]{2}{*}{$\mathrm{gCC}$} & 24 & Group & 2.753 & 3.09 & .015 & 6.452 & 3.26 & .012 & 7.657 & 3.46 & .018 \\
\hline & & Age & 0.118 & -0.76 & NS & 0.277 & -0.63 & NS & 0.329 & -0.90 & NS \\
\hline \multirow[t]{2}{*}{ Left mCST } & 25 & Group & 0.557 & 3.25 & .015 & 2.334 & 3.03 & .015 & 4.137 & 3.61 & .023 \\
\hline & & Age & 0.024 & -1.18 & NS & 0.103 & -1.4 & NS & 0.182 & -1.46 & NS \\
\hline \multirow[t]{2}{*}{ Right mCST } & 25 & Group & 2.098 & 2.3 & .059 & 4.997 & 2.92 & .016 & 6.987 & 3.28 & .019 \\
\hline & & Age & 0.092 & -1.25 & NS & 0.2201 & -1.13 & NS & 0.308 & -1.21 & NS \\
\hline
\end{tabular}

Note:-NS indicates not significant; SE, standard error; gCC, genu of the CC.

${ }^{a}$ All $P$ values have been corrected for multiple comparisons using the false discovery rate method.
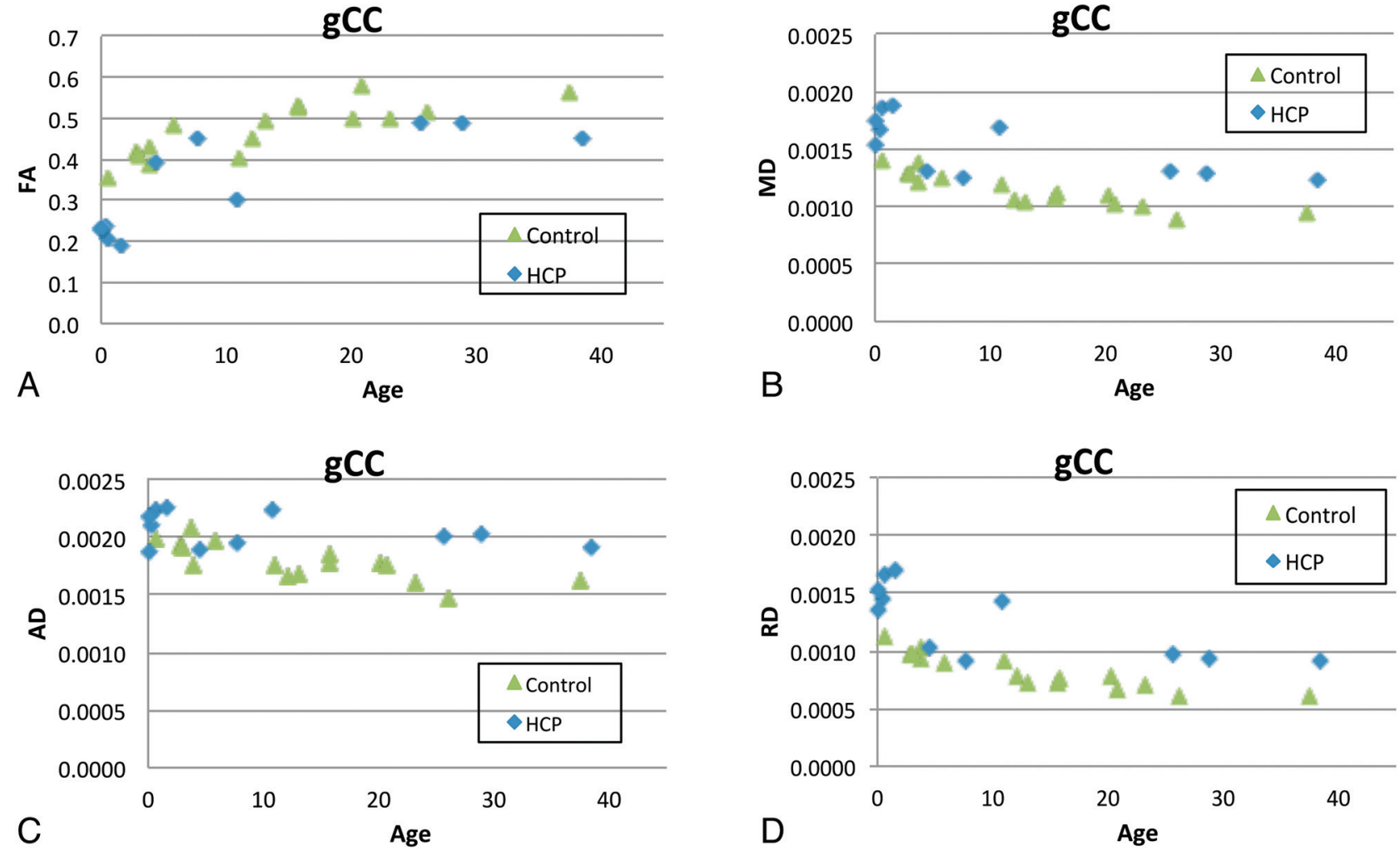

FIG 6. Comparison of DTI measures derived from PDT in the genu of the CC. A, FA, B, MD, C, AD. D, RD.

Table 3: Multiple regression analysis for each tract and DTI variable ${ }^{a}$

\begin{tabular}{|c|c|c|c|c|c|c|c|c|c|c|c|c|c|c|}
\hline & \multirow[b]{2}{*}{$d f$} & \multirow[b]{2}{*}{ Source } & \multicolumn{3}{|c|}{ FA } & \multicolumn{3}{|c|}{$M D$} & \multicolumn{3}{|c|}{$A D$} & \multicolumn{3}{|c|}{ RD } \\
\hline & & & SE & $T$ Value & $P$ & SE & $T$ Value & $P$ & SE & $T$ Value & $P$ & SE & $T$ Value & $P$ \\
\hline \multirow[t]{2}{*}{$\mathrm{gCC}$} & 24 & Group & 0.022 & 5.36 & .0024 & 0.000 & -6.41 & .0008 & 0.000 & -4.57 & .0005 & 0.000 & -6.57 & .0004 \\
\hline & & Age & 0.001 & 6.67 & .0012 & 0.000 & -4.62 & .0006 & 0.000 & -2.11 & .0523 & 0.000 & -5.33 & .0003 \\
\hline \multirow[t]{2}{*}{ Left mCST } & 25 & Group & 0.023 & 0.47 & NS & 0.000 & -3.74 & .0015 & 0.000 & -4.25 & .0005 & 0.000 & -3.18 & .0052 \\
\hline & & Age & 0.001 & 6.60 & .0003 & 0.000 & -4.62 & .0003 & 0.000 & -2.79 & .1080 & 0.000 & -5.25 & .0002 \\
\hline \multirow[t]{2}{*}{ Right mCST } & 25 & Group & 0.020 & 0.89 & NS & 0.000 & -4.26 & .0005 & 0.000 & -3.46 & .0028 & 0.000 & -2.91 & .0090 \\
\hline & & Age & 0.001 & 6.81 & .0002 & 0.000 & -5.26 & .0002 & 0.000 & -2.94 & .0088 & 0.000 & -5.19 & .0002 \\
\hline
\end{tabular}

Note:-NS indicates not significant; SE, standard error; gCC, genu of the CC.

${ }^{a}$ All $P$ values have been corrected for multiple comparisons using the false discovery rate method.

tracts examined in children with hydrocephalus after accounting for age. FA in the genu of the CC was also found to be significantly lower in the children with hydrocephalus. These tract-based findings are consistent with previously reported region of interestbased results in terms of the patterns of abnormality, ${ }^{5}$ and the differences in the patterns of DTI abnormalities between the genu of the CC and the mCST in the present study lend further credence to the concept of region-specific multifactorial variation in underlying white matter injury mechanisms in hydrocephalus. ${ }^{1,6}$
Although the group difference of FA in the left mCST and right mCST was not significant between the children with hydrocephalus and the controls (because some children with hydrocephalus had abnormally low FA while others had abnormally high FA values), the reduction in the connectivity measures in children with hydrocephalus remained statistically significant; this outcome emphasizes the importance of including both summary DTI measures and connectivity measures in future studies. 
Differences in the normalized connectivity index between the children with hydrocephalus and those in the control group in the left and right mCSTs, illustrated in Fig $4 D$, - E, suggest that WM connectivity is subtly decreased in these long pathways in the patient group. This apparent reduction in the volume of WM with the highest connectivity might suggest an expectation or decreased sensorimotor function in children with hydrocephalus relative to the controls.

\section{Limitations}

We note that the success of the PDT method may depend on the tract of interest, especially when it is performed in brains with large deformations, such as those in children with hydrocephalus. Even though the tracts were carefully examined to avoid extending into ventricle areas and different threshold levels were tested to ensure the consistency of the methodology, partial volume effect is difficult to quantify and cannot be excluded as a potential factor that may bias the results. The attempt to track the splenium of the CC was not successful in 9 of 12 children with hydrocephalus; this outcome can be attributed to the significant damage or even agenesis of the area. Our initial attempt to track the whole corticospinal tract was unsuccessful, likely due to the severe distortion of anatomic structures, which may have caused imaging and modeling errors, accumulated over a long pathway, to exceed even a very lenient threshold set in the PDT. This study may also suffer from the limited sample size and the lack of pathologic evidence to support the predictive value of the PDT-based measures. Nevertheless, the findings in the present study represent an initial success in the application of PDT in analyzing WM abnormalities in pediatric hydrocephalus. The success also points to possible applications of this approach in patients with other pathologies that distort white matter tracts, such as brain tumors or tuberous sclerosis. Future studies should include a larger sample size and both short- and long-term follow-up so that we can investigate the longitudinal progression during the developmental stages of childhood.

\section{CONCLUSIONS}

It was demonstrated that the summary measures of connectivity and DTI based on the results of probabilistic diffusion tractography are sensitive to differences in WM microstructure in children with hydrocephalus compared with healthy controls. The PDT method may be a useful noninvasive tool to serve as an imaging biomarker for predicting behavioral and neuropsychological outcomes in this patient population with hydrocephalus or in assessing therapeutic efficacy in the treatment of hydrocephalus.

Disclosures: Akila Rajagopal—RELATED: Grant: NIH/NINDS IR01NS066932.* Joshua S. Shimony-RELATED: Grant: $\mathrm{NIH},{ }^{*}$ Comments: The study is part of an NIH grant. Robert C. McKinstry — RELATED: Grant: NIH, ${ }^{*}$ Comments: R01 NS066932 (W. Yuan, Principal Investigator; D.D. Limbrick, Washington University Principal Investigator) September 30, 2009 to September 30, 2014, Cincinnati Children's Hospital/NIH/ NINDS, Longitudinal DTI Study in Children Treated for Congenital Hydrocephalus, UNRELATED: Employment: I appeared in the Siemens PET/MRI commercial and advertisement campaign. This support had nothing to do with this manuscript, Payment for Lectures (including service on Speakers Bureaus): Siemens paid an honoraria, travel, meals, and lodging so that I could give lectures reporting our experience with the PET/MRI. These lectures had nothing to do with the manuscript. Thomas Maloney-RELATED: Grant: NIH/NINDS 1R01 NS066932.* Francesco T. ManganoRELATED: Grant: NINDS/NIH.* Scott K. Holland-RELATED: Support for Travel to Meetings for the Study or Other Purposes: NIH R01 grant,* Comments: Annual research group meeting for this project was held in St. Louis. The grant covered the travel and lodging costs for me and others involved in the meeting, UNRELATED: Grants/Grants Pending: NIH.* Sarah Simpson—RELATED: Grant: NIH, Comments:
The grant that supports this research pays my salary. Weihong Yuan-RELATED: Grant: NIH/NINDS 1R01 NS066932.* *Money paid to the institution.

\section{REFERENCES}

1. Yuan W, McKinstry RC, Shimony JS, et al. Diffusion tensor imaging properties and neurobehavioral outcomes in children with hydrocephalus. AJNR Am J Neuroradiol 2013;34:439-45

2. Rourke BP. Syndrome of Nonverbal Learning Disabilities. New York: Guilford; 1995

3. Mataró M, Junque C, Poca MA, et al. Neuropsychological findings in congenital and acquired childhood hydrocephalus. Neuropsychol Rev 2001;11:169-78

4. Del Bigio MR, Wilson MJ, Enno T. Chronic hydrocephalus in rats and humans: white matter loss and behavior changes. Ann Neurol 2003;53:337-46

5. Yuan W, Mangano F, Air EL, et al. Anisotropic diffusion properties in infants with hydrocephalus: a diffusion tensor imaging study. AJNR Am J Neuroradiol 2009;30:1792-98

6. Assaf Y, Ben-Sira L, Constantini S, et al. Diffusion tensor imaging in hydrocephalus: initial experience. AJNR Am J Neuroradiol 2006;27:1717-24

7. Hattori TI, Aoki S, Yuasa T, et al. White matter alteration in idiopathic normal pressure hydrocephalus: tract-based spatial statistics study. AJNR Am J Neuroradiol 2012;33:97-103

8. Oishi KF, Jiang H, Li X, et al. Atlas-based whole brain white matter analysis using large deformation diffeomorphic metric mapping: application to normal elderly and Alzheimer's disease participants. Neuroimage 2009;46:486-99

9. Hattingen EJ, Melber J, Blasel S, et el. Diffusion tensor imaging in patients with adult chronic idiopathic hydrocephalus. Neurosurgery 2010;66:917-24

10. Yuan W, Holland SK., Shimony, J, et al. Quality assurance in multiinstitution and multi-scanner MRI neuroimaging research. In: Proceedings of the International Paediatric Society London 2011: 6th Congress and Exhibition of the Joint Societies of Paediatric Radiology, London, UK. May 27-31, 2011

11. Behrens TE, Berg HJ, Jbabdi S, et al. Probabilistic diffusion tractography with multiple fiber orientations: what can we gain? Neuroimage 2007;34:144-55

12. Behrens TE, Woolrich MW, Jenkinson M, et al. Characterization and propagation of uncertainty in diffusion-weighted MR imaging. Magn Reson Med 2003;50:1077-88

13. Guye M, Parker GJ, Symms M, et al. Combined functional MRI and tractography to demonstrate connectivity of the human primary motor cortex in vivo. Neuroimage 2003;19:1349-60

14. Ciccarelli O, Behrens TE, Altmann DR, et al. Probabilistic diffusion tractography: a potential tool to assess the rate of disease progression in amyotrophic lateral sclerosis. Brain 2006;129:1859-71

15. Benjamini Y, Hochberg Y. Controlling the false discovery rate: a practical and powerful approach to multiple testing. J R Stat Soc 1995;57:289-300

16. Parker GJ, Haroon HA, Wheeler-Kingshott CA. A framework for a Streamline-Based Probabilistic Index of Connectivity (PICo) using a structural interpretation of MRI diffusion measurements. J Magn Reson Imaging 2003;18:242-54

17. Tuch DS, Belliveau JW, Wedeen VJ. A path integral approach to white matter tractography. In: Proceedings of the 16th Scientific Meeting and Exhibition of the International Society of Magnetic Resonance in Medicine, Toronto, Ontario, Canada. May 3-9, 2000;8:791

18. Jones DK, Pierpaoli C. Confidence mapping in diffusion tensor magnetic resonance imaging tractography using a bootstrap approach. Magn Reson Med 2005;53:1143-49

19. Del Bigio MR. Neuropathological changes caused by hydrocephalus. Acta Neuropathologica 1993;85:573-85

20. Ramnani N, Behrens TE, Johansen-Berg H, et al. The evolution of prefrontal inputs to the cortico-pontine system: diffusion imaging evidence from Macaque monkeys and humans. Cerebral Cortex 2006;16:811-18 